\title{
Versuche zum Nachweis von Gärungsenzymen im Tierkörper. Von
}

Beth und Hans Euler.

(Aus dem biochemischen Laboratorium der Universität Stockholm.)

(Der Redaktion zugegangen am 1. Juni 1916.)

Nachdem der eine von uns die Möglichkeit erwogen hat, daß die Zuckerspaltung im Tierkörper nicht durch Vermittlung eines einzigen Organes, sondern stufenweise in mehreren Organen verläuft und also jedesmal eine der Reaktionsphasen der Zuckerspaltung durchlaufen wird, ${ }^{1}$ ) haben wir unter anderem Versuche darüber angestellt, welche Organe die Spaltung der Brenztraubensäure bewirkt und also ein der Neubergschen Carboxylase nahestehendes oder mit ihr identisches Enzym enthält.

. Unsere diesbezüglichen Versuche sind keineswegs abgeschlossen, aber wir teilen einige unserer Ergebnisse hier mit, da der eine von uns für einige Zeit verhindert ist, die Untersuchung fortzusetzen.

Die Tatsachen, die wir mitteilen wollen, sind folgende:

Verteilt man ganz frische Leber von Rind fein und schlämmt $4 \mathrm{~g}$ davon in $20 \mathrm{ccm}$ einer Lösung auf, welche $0,7 \mathrm{~g}$ brenztraubensaures Natrium enthält, und setzt $1 \mathrm{ccm}$ Toluol zu, so tritt nach einiger Zeit Gasentwicklung ein. Diese Gasentwicklung rührt sicher von der Spaltung des Natriumpyrovinates her, was dadurch bewiesen wird, daß Parallelversuche, welche mit den gleichen Lebermengen und gleichem Toluolzusatz teils mit Wasser, teils mit 2,5\% \% iger Glukoselösung angestellt wurden, keine oder nur unbedeutende Gasentwicklung ergaben. Wir führen beispielsweise folgenden Versuch an:

Die Zahlen sind Mittelwerte aus je 2 Parallelversuchen; sie geben die Kubikzentimeter Gas an, welche unter den gewählten Versuchsbedingungen bei Atmosphärendruck über Quecksilber erhalten wurden.

1) Euler und Funke, Diese Zeitschr., Bd. 77, S. 488, 1916. 
$4 \mathrm{~g}$ Leber $+1 / 2 \mathrm{ccm}$ Tolnol.

\begin{tabular}{c|c|c|c}
\hline Stunden & $\begin{array}{c}+20 \mathrm{ccm} 3,5 \% \text { ige } \\
\text { Na-Pyrovinatlösg. }\end{array}$ & $\begin{array}{c}+20 \mathrm{ccm} 2,5 \% \text { ige } \\
\text { Glukoselosung }\end{array}$ & $\begin{array}{c}+20 \mathrm{ccm} \\
\text { Wasser }\end{array}$ \\
\hline 12 & 14,5 & 6 & 4,2 \\
18 & 22,5 & 7,2 & 4,7 \\
36 & 31 & 7,5 & 5 \\
48 & 32,7 & 7,5 & 5,2 \\
60 & 33 & 7,5 & 5
\end{tabular}

Drei weitere, gleichartige Versuchsreihen mit entsprechenden Parallelversuchen gaben ganz gleichartige Resultate.

Die Deutung dieser Versuche wird dadurch erschwert, daß Brenztraubensäure, bezw. ihr Alkalisalz von vielen Bakterien unter Gasentwicklung gespalten wird, wie zuerst Karczag und seine Mitarbeiter ${ }^{1}$ ) in einer Reihe interessanter Arbeiten gezeigt haben.

$\mathrm{Da} B$ in oben angegebener Gasentwicklung auch Bakterien mitgewirkt haben, wird dadurch nahegelegt, da $\mathrm{B}$ das Gas bei den Versuchen mit Pyrovinat nur zur Hälfte aus $\mathrm{CO}_{2}$ bestand, während der Rest im wesentlichen als Wasserstoff angesprochen werden konnte, entsprechend den Ergebnissen von Karczag.

Da wir bei unseren Versuchen stets relativ bedeutende Mengen Toluol den Lösungen zugesetzt hatten - für genügende Mischung wurde stets gesorgt -, so geht aus unseren Versuchen hervor, daß die unser Material infizierenden Bakterien auch in Gegenwart von Toluol ihre spaltende Wirksamkeit auf Brenztraubensäure ausüben, wie dies Neuberg an Hefe bereits vor längerer Zeit konstatiert hat.

Es frägt sich nun, ob die gesamte Gasentwicklung von der bakteriellen Spaltung herstammt.

Wir sehen zur definitiven Beantwortung dieser Frage zwei Möglichkeiten: Die eine besteht in Experimenten, welche unter Ausschluß wirksamer Bakterien angestellt werden; derartige Arbeiten sind aber zweifellos mit erheblichen Schwierigkeiten verknüpft. Die andere besteht im Studium der im betreffenden tierischen Material vorkommenden Bakterien und ihres Verhaltens zu antiseptischen Mitteln.

1) Karczag und Mitarbeiter, Biochem. Zeitschr., Bd. 55, S. 79, 1913; Bd. 70, S. 317 u. 325, 1915. 
Versuche zum Nachweis von Gärungsenzymen im Tierkörper. 313

Wir haben uns einstweilen darauf beschränken müssen, nachzuweisen, daß bei analogen Versuchen, bei welchen Leber durch andere tierische Organsubstanz ersetzt wird, eine der obigen entsprechende Entwicklung von $\mathrm{CO}_{2}$ nicht eintritt.

$4 \mathrm{~g}$ zermahlene Niere $+1 \mathrm{ccm}$ Toluol. $37^{\circ}$.

\begin{tabular}{|c|c|c|c|c|c|c|}
\hline \multirow{2}{*}{ Stunden } & \multicolumn{3}{|c|}{$\begin{array}{c}20 \mathrm{ccm} \mathrm{3,5} \% \text { ige Na-Pyrovinat- } \\
\text { lösung }\end{array}$} & \multicolumn{3}{|c|}{$20 \mathrm{ccm}$ Wasser } \\
\hline & 1. Versuch & 2. Versuch & 3. Versuch & 1. Versuch & 2. Versuch & 3. Versuch \\
\hline 1,5 & 1,5 & 1,5 & 0,5 & 0,5 & 0,5 & 0,5 \\
\hline 5 & 5,5 & 8,5 & 1,5 & 1,5 & 0,5 & 1,0 \\
\hline 22 & 7 & 27 & 3 & 2,0 & 2,0 & 2,0 \\
\hline 31 & 8,5 & 35 & 3 & 2,0 & 2,0 & 2,0 \\
\hline 46 & 8 & 40 & 2,5 & 1,8 & 1,8 & 2,0 \\
\hline
\end{tabular}

Beim zweiten Versuch war offenbar eine ungewöhnlich starke Bakterieninfektion eingetreten. Indessen ist hervorzuheben, daß höchstens $2 \mathrm{ccm} \mathrm{CO}_{2}$ bei diesem Versuch entwickelt wurden. Die $\mathrm{CO}_{2}$-Entwicklung bei den Parallelversuchen 1 und 3 mit Pyrovinat war minimal.

In ähnlicher Weise wie Niere scheint sich Pankreas von Rind zu verhalten.

Es ergibt sich also zunächst, daß Brenztraubensäure in Gegenwart von Rinderleber in anderer Weise gespalten wird als in Gegenwart anderer Organe, wie Niere oder Pankreas.

Man könnte dies für einen Anhaltspunkt dafür ansehen, $\mathrm{da} \beta$ bei den Versuchen mit Leber neben der Bakterienspaltung gleichzeilig eine Spaltung durch ein der Leber eigentümliches Gärungsenzym, eine Carboxylase, eintritt.

Einen Beweis hierfür liefern, wie wir betonen wollen, unsere bis jetzt ausgeführten Versuche nicht. Es ist nämlich keineswegs ausgesch'ossen, daß mit der Leber andere Bakterien in die Pyrovinatlösung eingeführt wurden, als mit Niere und Pankreas, und zwar Bakterien, welche aus Brenztraubensäure $\mathrm{CO}_{2}$ abzuspalten imstande sind.

Anderseits halten wir die gewonnenen Ergebnisse für interessant genug, um uns weiter eingehend mit der Verfolgung dieser Versuche zu beschäftigen. 